\title{
Editorial
}

\section{MicroRNAs meet cell death}

\author{
G Melino $^{*, 1,2}$ and RA Knight ${ }^{*, 1}$ \\ Cell Death and Differentiation (2010) 17, 189-190; doi:10.1038/cdd.2009.122
}

Micro RNAs (miRs) are a family of small noncoding (nc) RNAs, which, similar to other small ncRNA families, such as siRNAs and Piwi RNAs, add a further level of complexity to the regulation of gene expression. miRs represent between 1 and $3 \%$ of all 'genes', although ncRNAs probably cannot be strictly called 'genes'. Since their identification in Caenorhabditis elegans in 1993, similar molecules have now been described across the species spectrum, from bacteria (reviewed in Gottesman $^{1}$ ) and viruses ${ }^{2}$ to plants (reviewed in Mallory and Vaucheret $^{3}$ ) and animals. Although it has been estimated that roughly $30 \%$ of the transcriptome is regulated by miRs, this figure is largely derived from bioinformatics analysis, which can result in both false-positive and false-negative results, and different algorithms may provide only partially overlapping sets of mRNA transcripts that are theoretically regulated by any given miR. The importance of ncRNAs was recognized by the award of the Nobel prize in 2006, and CDD had the honour of hosting the corresponding Nobel lectures. ${ }^{4-8}$ In this issue, we focus more specifically on the role of miRs in differentiation and in cell death.

miR-encoding genes are transcribed by RNA polymerase II to yield pri-miRs, which are then processed by a nuclear RNase III enzyme, Drosha, to form stem-loop-structured premiRs. These are, in turn, transported to the cytosol, in which another RNase III enzyme, Dicer, cleaves off double-stranded portions of the hairpin, generating a short-lived dsRNA of circa 20-25 nucleotides. The duplex is unwound and one strand constitutes the mature miR, which is incorporated into miRprotein complexes (miRNPs). miRs guide miRNPs to binding sites in the $3^{\prime}$-UTR of target mRNAs, although complete complementarity between the 6-7 residues of the miR seed sequence and the UTR is not required for functional effects. Bound miRNPs mostly inhibit translation or destabilize target mRNAs (see below), resulting in the downregulation of encoded proteins. The crucial role of miRs in development and death, suggested by their long evolutionary pedigree, is reinforced by the observation that mice deficient in Dicer, and which therefore cannot form mature miRs, die as early as day E7.5. The molecular biology of miRs has been reviewed elsewhere in greater depth. ${ }^{9-12}$

But how do miRs affect differentiation? The role of miRs in one differentiation process, the maturation of the epidermis, is reviewed here by $\mathrm{Yi}$ and Fuchs. ${ }^{13}$ Normally, the mitotic plane of cells in the epidermal basal layer is aligned either horizontally (representing replication of the stem cell pool) or vertically (representing upward differentiation), and there is persuasive evidence that maintenance of the stem cell pool requires $\Delta$ Np63. ${ }^{14}$ Conditional knockout of Dicer under the control of an epidermal basal layer-specific promoter disrupts this process, and results in a severe hair phenotype similar to that of p63 knockout mice. ${ }^{15} \mathrm{Yi}$ and Fuchs ${ }^{13}$ show that a miR, miR-203, which is evolutionarily conserved at least from zebrafish to man, is dialectically antagonistic to $\Delta \mathrm{Np} 63$, and as the $\Delta \mathrm{Np63}$ expression declines during differentiation, so does the miR-203 expression increase. Indeed, miR-203 directly reduces $\Delta \mathrm{Np} 63$ expression. ${ }^{13,16-18}$ Thus, the reduction in $\Delta$ Np63 by miR-203 allows cells to exit the stem cell pool and to commence upward differentiation. Consistent with this, the miR-203 expression is dysregulated in psoriasis, a disorder of abnormal epidermal proliferation.

In keeping with the potential regulation of stemness by the miR-203-p63 pathway and its effects on cell proliferation, miR-203 is highly expressed in head and neck squamous-cell carcinomas and in T-cell lymphomas. Thus, miR-203 directly inhibits the expression of the BCR-ABL1 oncogene, and in many haematological malignancies, the chromosomal region containing the miR-203 sequence is lost or the promoter is hypermethylated, allowing an aberrant BCR-ABL1 expression. ${ }^{19} \mathrm{Yi}$ and Fuchs ${ }^{12}$ also discuss the role of miR-200 and miR-205, both highly expressed in the skin and in cancer cells. Here, the target mRNAs are the transcriptional repressors of E-cadherin, ZEB1 and ZEB2, thus promoting the expression of E-cadherin and affecting the epithelial-mesenchymal transition.

Continuing the stem cell theme, Godlewski et al. ${ }^{20}$ review the role of miRs in glioblastoma, a tumour thought to be derived from neural stem cells. Indeed, the miR profiles of neural stem cells and glioblastoma cells are strikingly similar. In glioblastoma, expression of miR-124 (which normally comprises $25 \%$ of total brain miRs) is reduced, allowing an increased expression of Cdk6 and continuous cell-cycle progression. Dysregulated expression of other miRs in glioblastoma, such as that of miR-21, also promotes proliferation by affecting the PI3 kinase pathway. As well as offering a further dimension to our understanding of this devastating cancer, knowledge of $\mathrm{miR}$ regulation of neural stem cells may advance our ability to manipulate stem cells for the treatment of neurodegenerative disease.

\footnotetext{
${ }^{1}$ Medical Research Council, Toxicology Unit, Hodgkin Building, Leicester University, Lancaster Road, PO Box 138, Leicester LE1 9HN, UK and ${ }^{2}$ Department of Experimental Medicine, University of Rome 'Tor Vergata', Rome 00133, Italy

*Corresponding authors: G Melino or RA Knight, MRC Toxicology Unit, Hodgkin Building, Leicester University, Lancaster Road, PO Box 138, Leicester LE1 9HN, UK. Tel: + 4439062042 7299; Fax: + 4439062042 7290; E-mail: gm89@le.ac.uk or r.knight@ich.ucl.ac.uk
} 
The role of miR-10 in development and cancer is reviewed by Lund. ${ }^{21}$ The surprising finding here is that miR-10, by binding a group of ribosomal protein transcripts containing a terminal oligo-pyrimidine motif, activates their translation by around $30 \%$, rather than more conventionally producing translational repression. It remains to be seen how widespread this translational activation is, but the possibility that miRs can regulate translation in both directions is intriguing. miR-10 (and other miR) genes have been retained within the homeobox (HOX) locus, a cluster of evolutionarily conserved developmental regulators, which are particularly important for anterior-posterior patterning. The miRs within the HOX cluster regulate the expression of $\mathrm{HOX}$ cluster products, such as HOXA1, HOXA3 and HOXD10, and therefore have important roles in development. In addition, miR-10 is deregulated in a large variety of human cancers, ranging from hepatocellular carcinoma, pancreatic cancer, breast cancer, melanoma, CLL, AML to glioblastoma.

In cancer, of course, we need to understand why incipient tumour cells do not do the decent thing and kill themselves, and therapeutically, we want to find better ways of killing them. The first real hint that miRs were critically involved in tumorigenesis came in 2002, when Calin et al. ${ }^{22}$ identified miR-15a and miR-16-1 as potential genes involved in the pathogenesis of chronic lymphocytic leukaemia (CLL), as their coding sequences were contained within a $790 \mathrm{~kb}$ region of loss at 13q14.3 commonly deleted in CLL. Subsequently, the same group showed that both miRs caused a posttranscriptional repression of $\mathrm{Bcl}-2$, a gene relevant to the pathogenesis of CLL, as increased $\mathrm{Bcl}-2$ expression inhibits apoptosis. ${ }^{23}$ This clearly stimulated interest in the connection between miRs and cell death. Now, Aqeilan et al. ${ }^{24}$ recall this historical perspective and describe the identification of miR-15a/miR-16-1 targets such as BCL-2, CDC2, ETS1, JUN, MCL1, MSH2, PDCP6IP, RAB9B and WT1. They finally discuss therapeutic perspectives that, although still in their infancy, hold great promise as additional anticancer therapies. Other miRs target different points of the intrinsic and extrinsic apoptotic pathways, reviewed in Garofalo et al. ${ }^{25}$ Examples of miR apoptotic targets are TNF- $\alpha$ (miR125b), FADD/Rip (miR155), p27/p57 (miR-221, miR-222), p21 (miR-17-92), Bim (miR-17-92, miR 106b-25) and Mcl-1 (miR-29).

No discussion of cell death and cancer would be complete without a consideration of the role of the p53 family. As reviewed by Hermeking, ${ }^{26} \mathrm{miR}-34 \mathrm{a}$ and $\mathrm{miR}-34 \mathrm{~b} / \mathrm{c}$ are both induced by $\mathrm{p} 53$. Conversely, miR-34a is part of a positive feedback loop that leads to further activation of p53 through an inhibition of SIRT1, an NAD-dependent deacetylase that inhibits several pro-apoptotic proteins. miR-34a and miR-34b/ c cause a cell-cycle arrest at G1, inhibit proliferation and colony formation in soft agar and induce cellular senescence. Indeed, the miR-34 family can target several genes relevant for G1-arrest (CCND1, CCNE2, CDK4, CDK6, MET, c-MYC, $\mathrm{N}-\mathrm{MYC}$ and CDC25C), cell death (BCL-2, c-MYC and NOTCH1) and senescence (SIRT1, E2F3 and HMGA2). Christoffersen et al. $^{27}$ expand on these data by showing a p53-independent upregulation of miR-34a during oncogeneinduced senescence, mediated by the repression of MYC. After a constitutive activation of B-RAF in fibroblasts, miR-34a and miR-146a show a significant induction, in parallel with senescence, but mediated by the ETS family transcription factor, ELK1. ${ }^{27}$ This reinforces the fact that the system is promiscuous in that multiple factors can induce a given miR, which, in turn, can regulate multiple targets. Clearly, for miR-34, in view of their high homology, the other members of the p53 family, p63 and p73, are primary suspects. Conversely, p53 (and maybe p63 and p73) can participate in other regulatory pathways involving different miRs, and indeed we have already discussed the relationship between p63 and miR-203 above. In addition, hints that miR-34 may be involved in other functions have been presented at a recent meeting on miRs and the p53 family. ${ }^{28}$ Here, Spizzo et al. ${ }^{29}$ produced evidence that miR-145 and p53 cooperate in a death-promoting regulatory loop targeting oestrogen receptor alpha in human breast cancer. miR-145 is induced by $p 53$ and induces apoptosis through a p53-dependent mechanism. This suggests the concept that a miR may work in synergy with other factors, which are induced simultaneously.

In cell death research, as well as in differentiation, we are conditioned to give primacy to the role of proteins. How often, at meetings, have presentations of microarray data been met with the response - 'but it's the protein that does the job'. It is now clear that this is only part of the story, as the regulation of protein expression by ncRNAs, including miRs, shows. It is tempting to speculate that here we may be seeing a modern version of an ancestral manipulative interaction between members of an older RNA world and the newer protein upstarts, particularly as functional ncRNAs seem to have such an ancient pedigree. It is already apparent that this dialogue confers an additional dimension of plasticity and subtlety to biological outputs, both physiological and pathological. Although, clearly, more basic research is needed, we should be aware of the huge potential of manipulating the manipulators.

\section{Conflict of interest}

The authors declare no conflict of interest.

1. Gottesman S. Trends Genet 2005; $7: 399-404$

2. Pfeffer S et al. Science 2004; 304: 734-736.

3. Mallory AC, Vaucheret H. Nat Genet 2006; 38 (Suppl): S31-S36.

4. Melino G et al. Cell Death Differ 2007; 14: 1975-1976.

5. Kornberg RD. Cell Death Differ 2007; 14: 1977-1980.

6. Mello CC. Cell Death Differ 2007; 14: 1981-1984.

7. Kornberg RD. Cell Death Differ 2007; 14: 1989-1997.

8. Mello CC. Cell Death Differ 2007; 14: 2013-2020.

9. Ventura A, Jacks T. Cell 2009; 136: 586-591.

10. Bartel DP. Cell 2009; 136: 215-233.

11. Valencia-Sanchez MA et al. Genes Dev 2006; 20: 515-524.

12. Calin GA, Croce CM. Nat Rev Cancer 2006; 6: 857-866.

13. Yi R, Fuchs E. Cell Death Differ 2009; 17: 229-235.

14. Lechler T, Fuchs E. Nature 2006; 437: 275-280.

15. Yi R et al. Nat Genet 2006; 38: 356-362.

16. Yi R et al. Nature 2008; 452: 225-229.

17. Lena AM. Cell Death Differ 2008; 15: 1187-1195.

18. Aberdam D et al. Trends Biochem Sci 2008; 33: 583-591.

19. Bueno MJ et al. Cancer Cell 2008; 13: 496-506.

20. Godlewski J et al. Cell Death Differ 2009; 17: 221-228.

21. Lund AH. Cell Death Differ 2009; 17: 209-214.

22. Calin GA et al. Proc Natl Acad Sci USA 2002; 99: 15524-15529.

23. Cimmino A et al. Proc Natl Acad Sci USA 2005; 102: 13944-13949.

24. Aqeilan RI, Calin GA, Croce CM. Cell Death Differ 2009; 17: 215-220.

25. Garofalo M et al. Cell Death Differ 2009; 17: 200-208.

26. Hermeking H. Cell Death Differ 2009; 17: 193-199.

27. Christoffersen NR et al. Cell Death Differ 2009; 17: 236-245.

28. Sayan E et al. Cell Death Differ 2009; 17: 373-375.

29. Spizzo R et al. Cell Death Differ 2009; 17: 246-254. 